Abstract ID: 47

\title{
Correlation between schroeder-phase effect and distortion product otoacoustic emission findings
}

Nur Baizura Salahuddin | Sarah Rahmat

Department of Audiology \& Speech-Language Pathology, Kulliyyah of Allied Health Sciences, International Islamic University Malaysia

Introduction: Schroeder-phase masking has been proven to be more sensitive than pure tone audiometry in detecting changes in cochlear function. Schroeder harmonic complexes with different phases have been observed to excite basilar membranes differently and give different masking abilities ('phase effect') when used as maskers. Previous theory suggested that phase effect was contributed by cochlear non-linearity of outer hair cells (OHC); however the theory was derived from behavioral observation alone. Therefore, this study aims to further investigate the cochlear non-linearity involvement in phase effect mechanism by measuring the Schroeder phase effect together with another electrophysiological test that measures the cochlear non-linearity function, i.e. Distortion Product Otoacoustic Emission (DPOAE). Methods: Twelve normal hearing and four sensorineural hearing loss subjects were recruited. Schroeder phase masking test was conducted and phase effect (using $75 \mathrm{~dB}$ A masker) at $1 \mathrm{kHz}$ and $2 \mathrm{kHz}$ was measured. DPOAE was recorded at multiple intensities (45-75 dB SPL) for $1 \mathrm{kHz}$ and $2 \mathrm{kHz}$, and slope of DPOAE input output function was measured. Correlation analysis was performed to find correlation between phase effect and slope of DPOAE input output function. Results: Result showed no significant correlation $(p>0.05)$ between phase effect and slope of DPOAE input output function. Conclusions: This findings suggest that Schroeder-phase effect may not be/ may not only be contributed by OHC's cochlear non-linearity. This finding opens the possibility of other auditory functions' involvement in phase effect mechanism, and contribute to better understanding towards auditory perceptions.

KEYWORDS: Cochlear Non-linearity; Schroeder Phase Masking; phase effect mechanism 